\title{
MANIFESTATIONS OPHTALMOLOGIQUES AU COURS DU SYNDROME D'APERT : A PROPOS D'UN CAS
}

\author{
MA. EL AFRIT, H. MAZLOUT, S. TROJET, M. BOULADI, S. MNIF, H. MNASRI, M. TRIKI, A. KRAIEM \\ SERVICE D'OPHTALMOLOGIE. HÔPITAL HABIB THAMEUR. TUNIS
}

\begin{abstract}
RESUME
Introduction : Parmi les crâniosténoses, le syndrome d'Apert demande la collaboration de plusieurs spécialistes, pour sauver ce qui peut l'être de la fonction visuelle des patients et permettre un développement cérébral le plus proche de la normale.

Observation : Nous présentons le cas d'une jeune suivie et traitée depuis son jeune âge pour un syndrome d'Apert. Elle a subi plusieurs interventions successives pour garder à un âge assez avancé une fonction visuelle appréciable à 3/10.Les modifications anatomiques ont inéluctablement influé sur l'état visuel de la patiente avec une myopie forte,un astigmatisme relativement important et une atrophie papillaire partielle.

Discussion : D'origine génétique, le syndrome d'Apert est dû à une mutation allélique du récepteur 2 d'un facteur fibroblastique.

Les signes de souffrance cérébrale sont inévitables, et l'atrophie optique relative représente la séquelle fonctionnelle principale.

Conclusion : Une prise en charge de longue haleine est nécessaire dans le syndrome d'Apert pour espérer sauver une fonction visuelle utile.
\end{abstract}

Mots clés : crâniosténose, syndrome d’Apert, chirurgie de décompression, atrophie optique.

Introduction : Several craniosynostotic syndromes are described such as Apert syndrome in which collaboration between different specialists is necessary to preserve visual function and to allow normal cerebral development.

Case-report : It's a case note of a girl with Apert syndrome. She underwent since her infancy several surgical operations. Anatomic modifications affected her visual status with a best visual acuity of $3 / 10$, high myopia, astigmatism and partial optic atrophy.

Discussion : Apert syndrome is a genetic disorder due to a mutation in fibroblast receptor growth factor genes. Optic atrophy attributed to optic neuropathy represents the major functional sequella and the major cause of visual loss.

Conclusion : Apert syndrome, like all craniosynostotic syndromes, requires a correct management in order to preserve the visual function.

Key-Words : craniosynostosis, Apert syndrome, decompression surgery, optic atrophy.

\section{INTRODUCTION}

Le syndrome d'Apert est une acrocéphalosyndactylie caractérisée par une dysmorphie crânio-faciale avec une crâniosténose, une syndactylie aux mains et aux pieds et d'autres malformations cérébrales (1). C'est une affection rare le plus souvent transmise selon le mode autosomique dominant bien que des cas sporadiques existent (2). Le traitement chirurgical précoce a pour but de minimiser les effets délétères de l'hypertension intracrânienne par l'augmentation du volume crânien et d'améliorer la disposition des structures orbito-cérébrales.

Nous présentons le cas d'une patiente présentant un syndrome d'Apert ayant bénéficié d'une chirurgie crânio-faciale et qui présente actuellement une altération séquellaire de la fonction visuelle.

\section{OBSERVATION}

Nous présentons le cas d'une jeune fille âgée de 31 ans présentant un syndrome d'Apert. II s'agit d'un cas isolé, sans notion de consanguinité parentale ni de cas similaires dans la famille.

Cette patiente a subi deux interventions chirurgicales successives, d'abord dans la petite enfance : avancement de l'os frontal puis à l'âge de 4 ans avancement facial de type Lefort III.

Avec la croissance, le maxillaire supérieur ne se développant pas, une dégradation morphologique s'est progressivement installée avec l'apparition d'un exorbitisme, d'un recul du maxillaire supérieur avec béance et d'une étroitesse de l'arcade dentaire supérieure.

De ce fait, et à l'âge de 18 ans, une troisième intervention 
a été indiquée ; elle a consisté en un avancement facial avec bipartition.

La patiente s'est récemment présentée à notre consultation pour baisse récente de l'acuité visuelle aux 2 yeux.

L'examen ophtalmologique a noté une exophtalmie bilatérale, accompagnée d'un hypertélorisme, et de fentes palpébrales antimongoloïdes (figure 1). La réfraction était de type myopique (7 dioptries à l'œil droit et 8 dioptries à l'œil gauche) avec un astigmatisme régulier (4,5 dioptries à l'œil droit et 3,5 dioptries à l'œil gauche). L'acuité visuelle était limitée à 3/10 avec correction non améliorable aux 2 yeux. Par ailleurs, l'examen du segment antérieur de l'œil était sans particularités. L'examen du fond d'œil a objectivé une pâleur papillaire marquée aux deux yeux à la limite de l'atrophie optique et expliquant l'atteinte de la fonction visuelle (figure 2). L'examen du champ visuel était altéré aux 2 yeux avec de multiples scotomes absolus (figure 3 ). L'examen général était normal à part une syndactylie des doigts et des orteils.

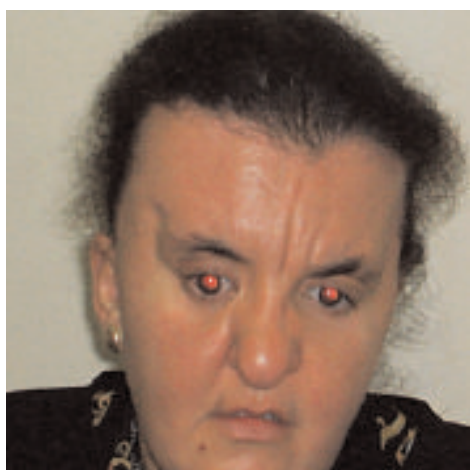

Fig. 1 : Syndrome d'Apert :exophtalmie

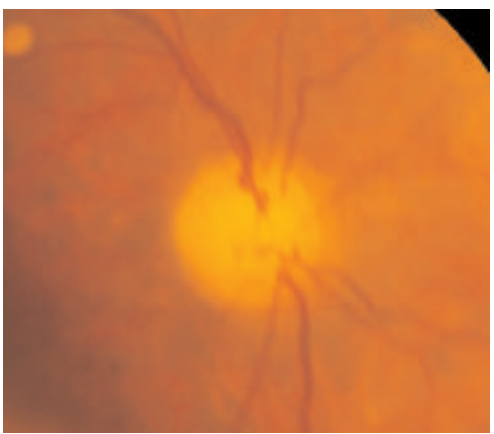

Fig. 2 : Photo du fond d'œil (oeil gauche): pâleur papillaire
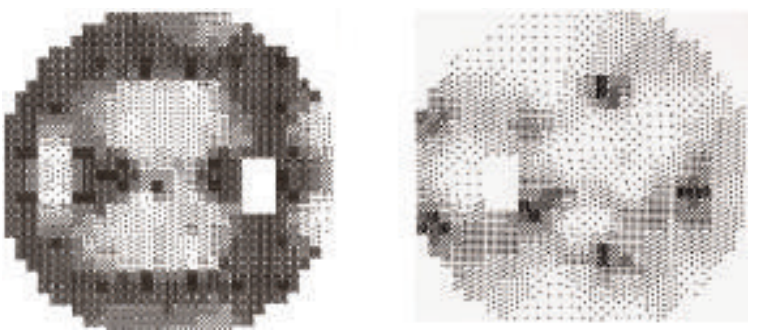

Fig. 3 : Champ visuel automatique: scotomes absolus.

\section{DISCUSSION}

Le syndrome d'Apert est l'un des plus fréquents syndromes de crâniosténoses (Crouzon, Pfeiffer, Saethrechotzen..). Sa prévalence est faible, estimée à 12,5 à 15,5 cas par million de nouveaux-nés vivants (3). Sur le plan génétique, le syndrome d'Apert est dû à une mutation allélique du récepteur 2 du facteur de croissance fibroblastique (FGFR2) (4).

La morbidité oculaire au cours du syndrome d'Apert résulte d'une disproportion entre le contenant (la boite crânienne) et le contenu (l'œil et le cerveau) qui continue de croître (3). La fermeture prématurée de plusieurs sutures crâniennes entraîne un rétrécissement des volumes crânien et orbitaire (3). Ainsi, l'orbite devient incapable de contenir et de protéger correctement l'œil avec comme conséquences un hypertélorisme, et un strabisme (5). L'altération de la fonction visuelle est la complication la plus sévère, elle est assez fréquente au cours du syndrome d'Apert (54 à $73 \%$ ) (3).

Elle peut être dûe à une kératite d'exposition, à des cicatrices cornéennes, à une amblyopie ou à une atrophie optique [5]. L'amblyopie, définie comme la baisse de l'acuité visuelle d'un œil par rapport à l'autre, est réputée de loin la cause la plus fréquente de détérioration visuelle. D'origine strabique, elle peut être imputée à la chirurgie crânio-faciale ; celle-ci modifiant l'alignement oculaire [3].

Quant à notre patiente, elle aurait présenté une neuropathie optique d'origine compressive ou secondaire à une hypertension intracrânienne prolongée avant la réalisation de la chirurgie de décompression. Elle peut également représenter une complication de cette chirurgie. Cependant, nous ignorons le statut visuel de la patiente avant et après les différentes chirurgies réalisées.

L'incidence de l'atteinte du nerf optique dans les crâniosténoses varie de 54 à 100\% [6] [7]. Cette incidence a nettement baissé avec les progrès de la chirurgie crânio faciale [3].

Actuellement le traitement et le protocole chirurgical de cette affection sont bien codifiés. En effet, la chirurgie comporte 3 temps [8] : Le premier temps consiste en un avancement fronto-orbitaire au cours de la première année de la vie afin d'élargir la base crânienne, de protéger l'orbite supérieur et de décomprimer le cerveau par des craniectomies latérales et postérieures. Le second temps réalisera un avancement facial entre 1 et 10 ans. Le troisième temps à la fin de la croissance comportera une chirurgie faciale définitive en vue d'un résultat plus esthétique.

Il faut insister sur la nécessité d'un bilan ophtalmologique soigneux avant et après la chirurgie. 


\section{CONCLUSION}

La détérioration visuelle est fréquente au cours du syndrome d'Apert. L'atrophie optique était la cause la plus fréquente avant l'ère de la chirurgie crânio faciale. L'incidence de cette complication est en baisse avec les nouveaux protocoles chirurgicaux mais une prise en charge patiente et de longue haleine est nécessaire pour un développement neurosensoriel sans dommage.

\section{REFERENCES}

1. Yacubian-Fernandez A et al. Le syndrome d'Apert : évaluation des malformations cérébrales et des altérations de la conformation déterminées par le traitement chirurgical. JFO $2004 ; 31: 2$ : 116-22.

2. Doutetien $\mathrm{C}$., Laleye $\mathrm{A}$. et al. Le syndrome d'Apert : à propos d'une observation. JFO $2003 ; 26,7: 738-42$

3. Khong J.J. et al. Ophthalmic findings in Apert's syndrome after craniofacial surgery. Ophthalmology 2006; 113: 347-52.

4. Wilkie AO. Craniosynostosis: genes and mechanisms. Hum Mol Genet 1997; 6:1647-56.

5. Buncic JR. Ocular aspects of Apert syndrome. Clin Plast Surg 1991; 18: 3159.
6. Friedenwald $\mathrm{H}$. On optic nerve atrophy associated with cranial deformity. Arch Ophthalmology 1901; 30:405-13.

7. Pemberton JW. et al. Craniosynostosis. A review of experience with forty patients with particular reference to ocular aspects and comments on operative indications. Am J Ophthalmol 1962; 54: 641-50.

8. David DJ. Advances in the management of the craniosynostosis. ANZ J Surgery 2003; 73: 949-57. 